\title{
Editorial
}

\section{Acute aortic syndrome}

Although the chest pain of acute aortic dissection is widely recognised, less consideration has been given to pain associated with other aortic pathologies. In light of contemporary concepts in aortic pathology we would like to present the pathology of a new cardiovascular syndromeacute aortic syndrome (AAS). ${ }^{1}$

This syndrome embraces a heterogeneous group of patients with a similar clinical profile that includes penetrating atherosclerotic aortic ulcer, intramural aortic haematoma, and the classic aortic dissection (fig 1). The physiopathological mechanism that precipitates the appearance of each of these entities is different. However, occasionally some patients exhibit several or all of these lesions, demonstrating the existence of a link between them. In such cases it is difficult to know which was the initiating event.

AAS is characterised clinically by aortic pain in a patient with a coexisting history of hypertension. In acute coronary syndromes, the existence of a typical chest pain that, since Heberden, has been called angina pectoris is well recognised. Likewise, in AAS chest pain is characteristic and has been called "aortic pain". The recognition of pain associated with these progressive aortic lesions is of paramount importance. A severely intense, acute, searing or tearing, throbbing, and migratory chest pain denotes that the patient may have an AAS. Anterior chest, neck, throat, and even jaw pain is related to involvement of the ascending aorta, whereas back and abdominal pain more often indicates that the affected segment is the descending aorta. ${ }^{2}$ The chest pain and clinical presentation of patients with penetrating aortic ulcer and intramural aortic haematoma is similar to that of classic aortic dissection. Severe, acute chest pain consistent with AAS may occur in patients with pronounced aortic root dilatation but without histopathologic evidence of aortic lesions. Aortic root stretching and distension are likely mechanisms for the pain experienced by these patients. Aortic pain may be confounded with that of ischaemic syndromes. Laboratory tests (creatine kinase and troponin), electrocardiographic changes, and chest $x$ ray may help to differentiate them. It

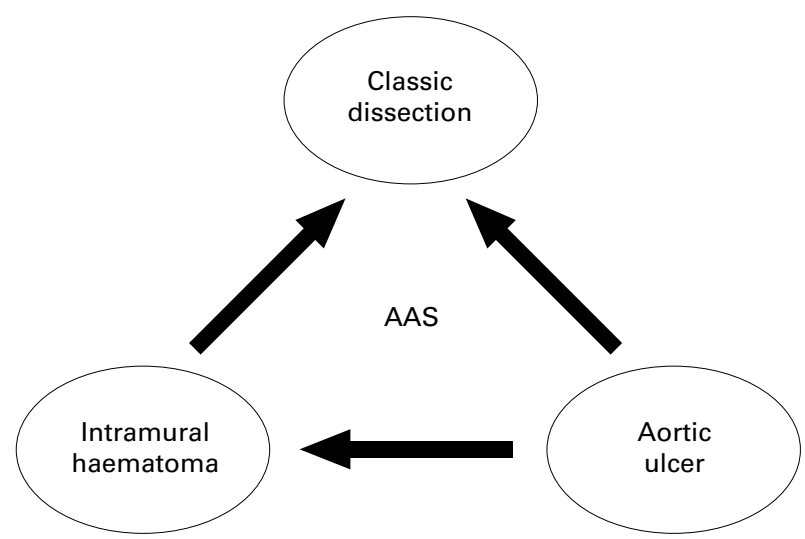

Figure 1 Acute aortic syndrome (AAS). Arrows indicate the possible progression of each of these aortic lesions.

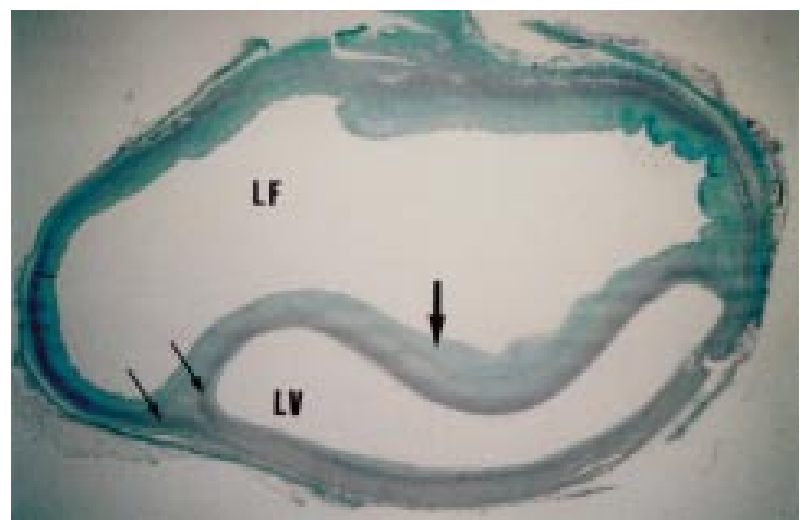

Figure 2 Histological section (Mason's technique) from a patient with aortic dissection. Muscle is stained in red and collagen in green. The aortic media (stained in red) is partitioned in two (arrows); one forms part of the dissection flap, the other forms the outer wall of the false channel. Large arrow indicates the dissection flap. LF, false lumen; LV, true lumen.

is also worth mentioning that acute coronary syndromes may be associated with, or result from, AAS.

Moderate to severe hypertension is a universal risk factor for the development of AAS. Inheritable disorders of elastic tissues also predispose to the development of classic aortic dissection, but hypertension is the most frequently associated condition. Hypertension is also the most common comorbid disease associated with penetrating aortic ulcers and intramural aortic haematomas. ${ }^{45}$

Several imaging techniques have been used in the detection and assessment of patients with AAS. Computed tomography (CT), magnetic resonance (MR), and transoesophageal echocardiography (TOE) are able to obtain echotomographic images that help to elucidate the pathoanatomy of this particular syndrome.

Although no pathogenetic mechanism is common to all aortic dissections, classic aortic dissection frequently exhibits the presence of an intimomedial flap and an entrance tear (figs 2 and 3). ${ }^{6}$ At pathology, aortic dissection is characterised by a separation of the aortic media of variable longitudinal and circumferential extension. The outer part of the aortic media will form with the adventitia the false channel outside wall, whereas the rest of the aortic media will constitute with the intimal layer to form the intimomedial flap (fig 2). ${ }^{7}$ The so called intimal flap is a misnomer because the flap tissue is composed mainly of aortic media delaminated from the aortic wall. This anatomical fact-the partition of the aortic media-is responsible for the two most important echotomographic signs in the diagnosis of aortic dissection: the existence of an aortic flap and the presence of a double channel aorta (fig 4). Most patients with dissection will have an entrance tear (fig 3). This intimomedial tear is the genesis of the splitting up of the aortic media. Thereafter, blood under pressure dissects the media longitudinally and, as a consequence, a double channel aorta is formed. The entrance tear is, in general, perpendicular to the long axis of the aorta and, therefore, it will be mainly detected by using axial or transversal echotomographic planes (fig 4). 


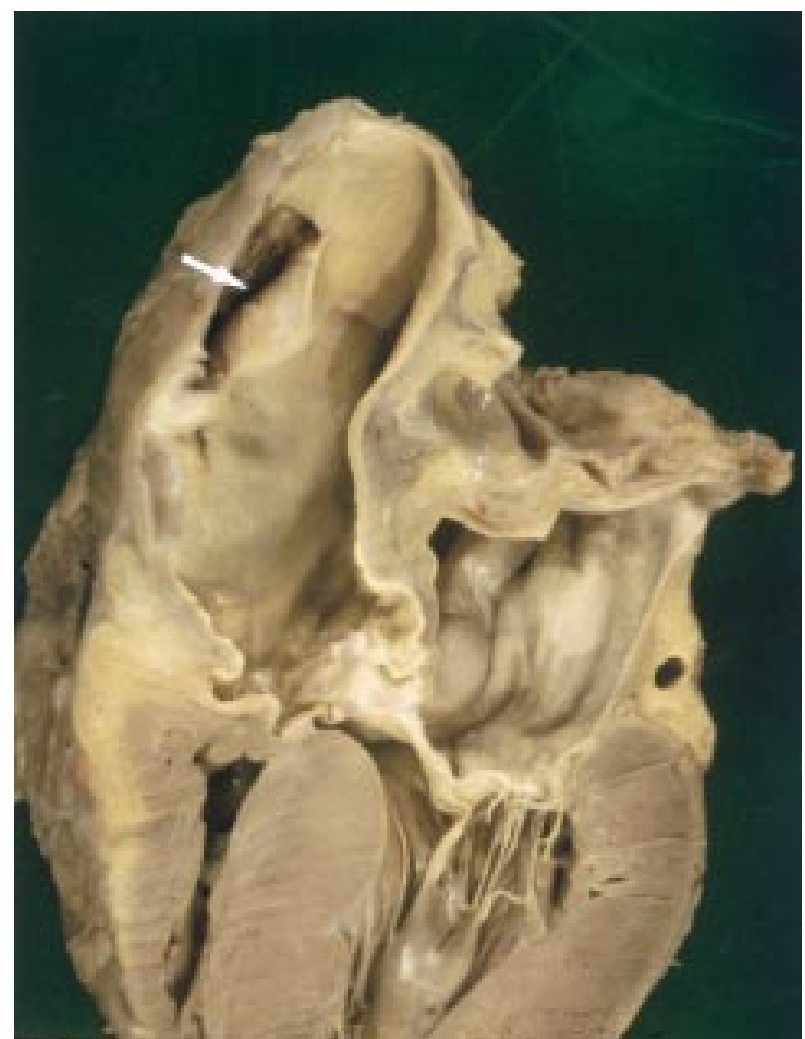

Figure 3 Anatomic specimen from a patient with type $A$ aortic dissection. The entrance tear in the ascending aorta is clearly visible (arrow).

In classic aortic dissection the entrance tear will be most frequently found at the areas of greatest hydraulic stress: the right lateral wall of the ascending aorta or in the proximal segment of the descending thoracic aorta. Many patients with classic aortic dissection will also have a re-entrance tear and several communicating points between the true and false lumen throughout the descending thoracic aorta. The re-entrance tear is usually located in the abdominal aorta, iliac arteries or other aortic branches, and it will be difficult to be visualised by any imaging technique. Bidirectional or unidirectional blood flow jets traversing the intimomedial flap along the descending thoracic aorta will be seen by TOE. These small communications are not re-entrance tears but the ostia of the intercostal or lumbar arteries that have been severed by the dissecting haematoma (fig 5). ${ }^{7}$ The differentiation between

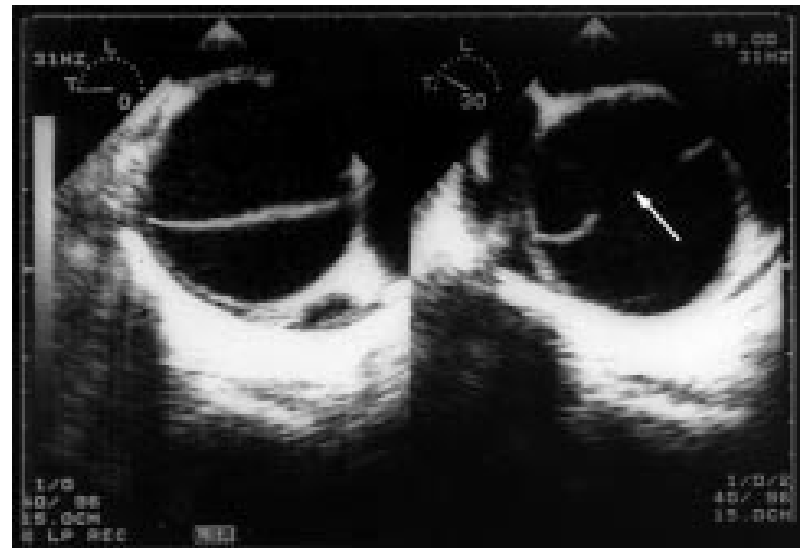

Figure 4 Transoesophageal echocardiographic study illustrating the double channel aorta and dissection flap (left panel), and the entrance tear (arrow) in the ascending aorta (right panel). Transversal planes. the true and false lumen has been considered an important aspect in the assessment of patients with dissection, and with the increasing use of endovascular repair it remains an important issue today. In classic aortic dissection, the true lumen tends to be smaller than the false lumen. Spontaneous contrast and some degree of thrombosis can be seen in the false lumen. In the true lumen, early laminar systolic flow is usually detected whereas in the false lumen diastolic, late systolic or slow swirling flow can be documented. ${ }^{8}$ Finally, aortic cobwebs, when detected, represent a pathognomonic sign of the false lumen.

Aortic intramural haematoma represents a novel variant of dissection characterised by the absence of an entrance tear (fig 6). ${ }^{5}{ }^{10-13}$ In these cases, the false lumen is created by a haemorrhage into the aortic media, most likely after rhexis of the vasa vasorum that penetrates the outer half of the aortic media from the adventitia and arborise at this level. In a few patients a tiny intimomedial entrance tear could be present but not visualised by the current imaging techniques. ${ }^{14}$ In these cases, the false channel (intramural haematoma) would not be decompressed by a re-entrance tear, and therefore there would be no possibility of detecting flow within the aortic wall. For an aortic intramural haematoma to develop, it is important that a re-entrance tear is absent. The entrance tear can be absent or, if present, it must be very small. ${ }^{14}$ This intramedial haemorrhage results in a circumferentially oriented blood containing space seen on echotomographic imaging studies (fig 7). On histologic analysis, a haematoma disrupting the aortic media is well documented (fig 8).

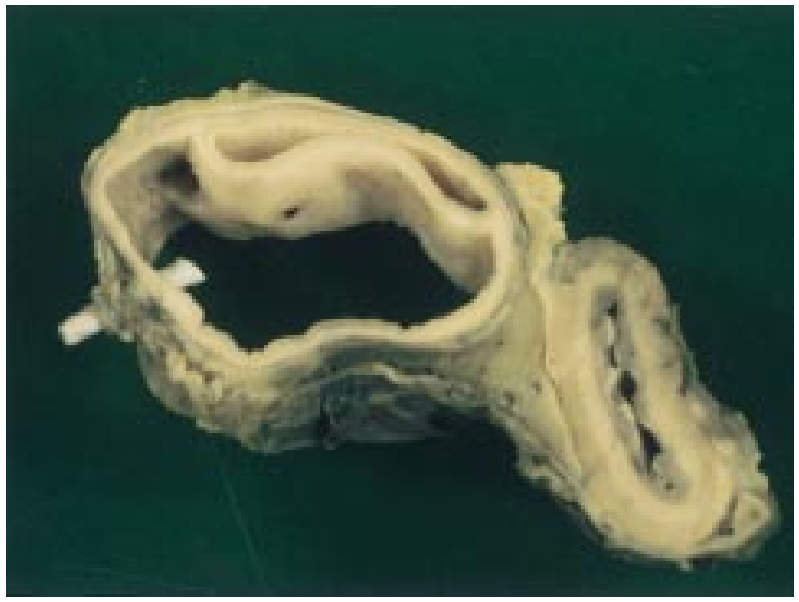

Figure 5 Anatomical cross section of the descending thoracic aorta. The ostium of an intercostal artery sectioned by the dissecting haematoma is clearly visible. A small hole in the dissection flap will permit blood flow between false and true aortic lumens.

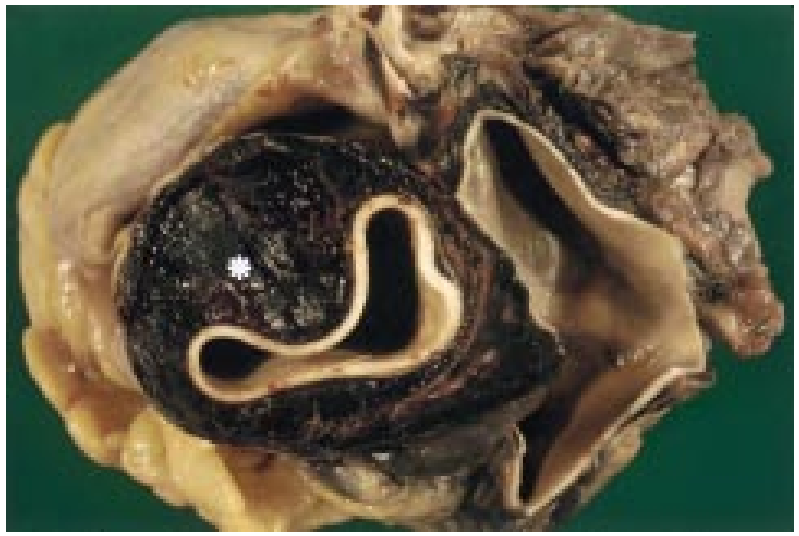

Figure 6 Anatomical cross section of the ascending aorta. An intramural aortic haematoma can be observed (asterisk). 

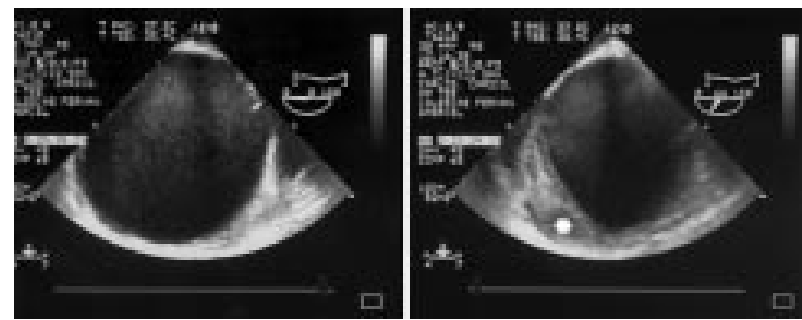

Figure 7 Transoesophageal echocardiographic study of a patient with a dilated aorta (left panel) and a crescent shaped thickening (asterisk) of the anterolateral wall of the aortic root that corresponds to an aortic intramural haematoma. Transverse planes.

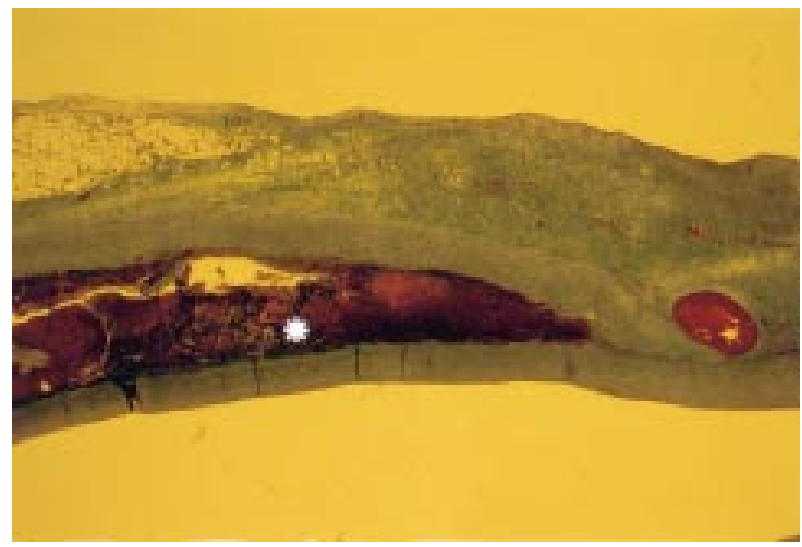

Figure 8 Histological section (Mason's technique) of a patient with intramural haematoma. Splitting of the aortic media by a haematoma (asterisk) is well documented.

In some patients, one can identify by TOE echolucent zones within the aortic wall thickening that probably represent areas of liquefaction of the intraparietal haematoma; when these areas are located immediately below the intimomedial flap, the dissection flap can be seen. ${ }^{5}$ Because there is no entrance tear, the intramedial haematoma does not communicate directly with the aortic lumen and, unlike the false lumen of classic aortic dissection, the presence of colour Doppler flow within the haematoma is an infrequent finding. For the same reason, this thickened aortic wall does not generally show enhancement with contrast administration on CT scanning, MR imaging, and angiography. Characteristically, aortic intramural haematoma displays dynamic behaviour, and thickened, crescent shaped or circular high attenuation areas along the aortic wall tend to vary with time..$^{51315}$ The mechanism of rapid regression of the aortic haematoma has not been elucidated. In some cases this segmental noncommunicating aortic dissection can progress to a typical dissection. ${ }^{51316}$ Increased permeability of the aortic wall leading to a pericardial, pleural, and mediastinal haemorrhage, and progression to an aortic rupture, has also been reported. ${ }^{5}$ It has been shown that aortic intramural haematoma may also originate from ulceration in an atherosclerotic aortic plaque ${ }^{14}{ }^{17}$; in these cases, the ulcerated aortic plaque behaves like an intimal tear.

The term "penetrating atherosclerotic aortic ulcer" describes the condition in which ulceration of an aortic atherosclerotic lesion penetrates the internal elastic lamina into the media ${ }^{1417}$ (fig 9). This lesion may precipitate intramural haemorrhage. In most patients this intramural (intramedial) haematoma is localised, but occasionally it can involve the entire descending thoracic aorta. ${ }^{17}$ These ulcers may be complicated by aneurysm formation ${ }^{18}$ (aortic remodelling), by breaking through into the adventitia to form a pseudoaneurysm, ${ }^{1}$ by rupturing completely (transmural rupture) into the mediastinum or the right and left

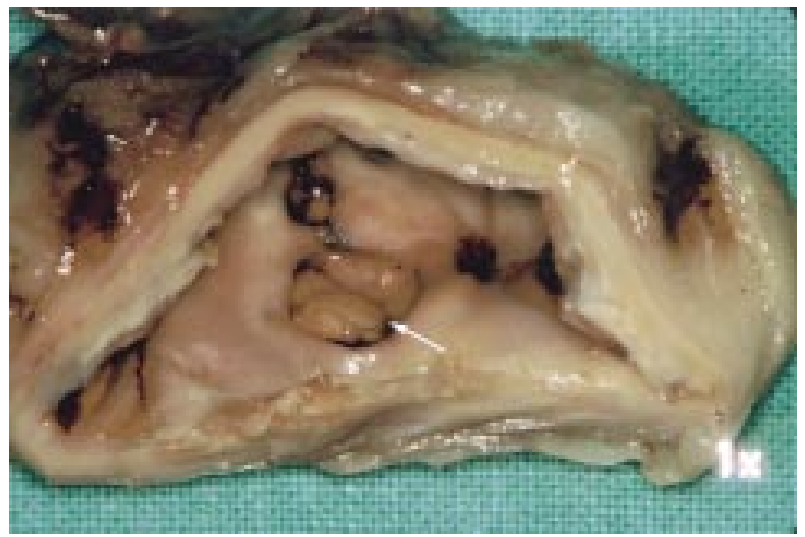

Figure 9 Anatomical cross section of the descending thoracic aorta. A penetrating atherosclerotic aortic ulcer is indicated by an arrow.

hemithorax, ${ }^{19}{ }^{20}$ and even by precipitating an aortic dissection. ${ }^{12122}$ The diagnosis of these ulcers is made on CT scan, MR imaging, and TOE by demonstration of an outpouching of the aortic wall with jagged edges, usually in the presence of extensive aortic atheroma ${ }^{13-25}$ (fig 10). Concomitant aneurysms of the descending aorta are commonly found. ${ }^{4}$

The absence of an ulcer crater distinguishes intramural aortic haematoma from penetrating aortic ulcers with intraparietal haemorrhage. Aortic ulcers are usually focal lesions most frequently located in the descending thoracic aorta. On the contrary, intramural haematoma and classic aortic dissection are more extensive lesions. In contradistinction to classic dissection, aortic dissection secondary to an aortic ulcer is shorter in extension, away from classic entrance tears, and has a thicker, calcified and static flap ${ }^{1}$ (fig 11). This type of dissection, in an area of gross atherosclerosis, is usually limited by neighbouring fibrosis and calcification. ${ }^{1}$

The clinical progress of these patients is unpredictable and, in many cases, unfavourable. Therefore, an early diagnosis is essential. Patients presenting with suspected AAS should be evaluated using non-invasive diagnostic modalities, and probably echocardiography should be performed initially. ${ }^{26}$ From a surgical and prognostic standpoint, this syndrome can be divided into two groups depending on the aortic segment involved: if the affected segment is the ascending aorta and/or the aortic arch-proximal AAS; if the involved segment is the descending aorta-distal AAS.

We reviewed the broad spectrum of acute aortic pathology focusing on the distinctive findings of each entity, and we have grouped them together under the name of AAS, with the intent of increasing clinical awareness of acute

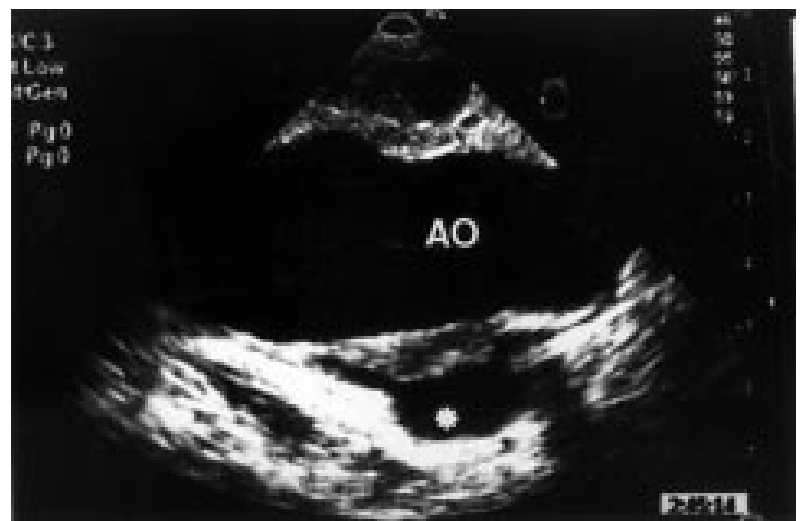

Figure 10 Transoesophageal echocardiographic study of a patient with an aortic ulcer (asterisk) in the descending thoracic aorta (AO). Longitudinal plane. 


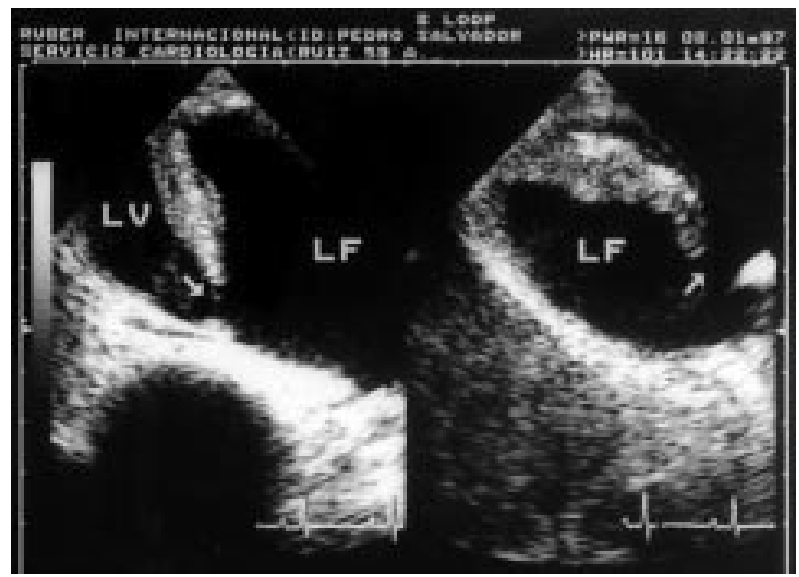

Figure 11 Transoesophageal echocardiographic scans in a patient with a limited acute aortic dissection secondary to aortic ulceration. A thick, calcified, irregular flap can be seen. Longitudinal planes. Arrow, entrance tear, LF, false lumen, LV, true lumen.

aortic lesions. We want to emphasise that these two variants of acute aortic pathology - intramural aortic haematoma and penetrating aortic ulcers - occur more frequently than previously thought. ${ }^{27}$ Increased attention to the different facets of AAS will lead to a more rapid diagnosis and an appropriate treatment of these patients.

ISIDRE VILACOSTA

Department of Cardiology,

Hospital Universitario de San Carlos,

C/ Serrano 46,

28001 Madrid, Spain

ivilac@medynet.com

Hospital Universitario de Valladolid

JOSÉ ALBERTO SAN ROMÁN

Spain

1 Vilacosta I, San Román JA, Aragoncillo P, et al. Penetrating atherosclerotic aortic ulcer: documentation by transesophageal echocardiography. $\mathcal{F} \mathrm{Am}$ Coll Cardiol 1998;32:83-9.

2 Wooley CF, Sparks EH, Boudoulas H. Aortic pain. Prog Cardiovasc Dis 1998;40:563-89.

3 Hirst EA Jr, Johns VJ Jr, Kime SW Jr. Dissecting aneurysm of the aorta: a review of 505 cases. Medicine 1958;37:217-79.

4 Coady MA, Rizzo JA, Hammond GL, et al. Penetrating ulcer of the thoracic aorta: What is it? How do we recognize it? How do we manage it? $\mathcal{F}$ Vasc Surg 1998;27:1006-16.
5 Vilacosta I, San Román JA, Ferreirós J, et al. Natural history and serial morphology of aortic intramural hematoma: a novel variant of aortic dissection. Am Heart f 1997;134:495-507.

6 Roberts WC. Aortic dissection: anatomy, consequences, and causes. $\mathrm{Am}$ Heart F 1981;101:195-214.

7 Vilacosta I, Castillo JA, San Román JA, et al. New echo-anatomical correlations in aortic dissection. Eur Heart F 1995;16:126-8.

8 Mohr-Kahaly S, Erbel R, Rennollet H, et al. Ambulatory follow-up of aortic dissection by transesophageal two-dimensional and color-coded Doppler echocardiography. Circulation 1989;80:24-33.

9 Vilacosta I, San Román JA, Aragoncillo P, et al. Aortic cobwebs: an anatomic landmark of the false lumen in aortic dissection documented by transesophageal echocardiography [abstract]. Eur Heart f 1996;17:439.

10 Yamada T, Tada S, Harada J. Aortic dissection without intimal rupture: diagnosis with MR imaging and CT. Radiology 1988;168:347-52.

11 Robbins RC, McManus RP, Mitchell RS, et al. Management of patients with intramural hematoma of the thoracic aorta. Circulation 1993;88( $\mathrm{Pt} 2): 1-10$.

12 Mohr-Kahaly S, Erbel R, Kearney P, et al. Aortic intramural hemorrhage visualized by transesophageal echocardiography: findings and prognostic implications. 7 Am Coll Cardiol 1994;23:658-64.

13 Nienaber CA, von Kodolitsch Y, Petersen B, et al. Intramural hemorrhage of the thoracic aorta: diagnostic and therapeutic implications. Circulation 1995;92:1465-72.

14 Vilacosta I, Martín de Dios R, González Pinto A. Aortic intramural hematoma during coronary angioplasty: Insights into the pathogenesis of intramedial hemorrhage. 7 Am Soc Echocardiogr 2000;13:403-6.

15 Ohmi M, Tabayashi K, Moizumi Y, et al. Extremely rapid regression of aortic intramural hematoma. F Thorac Cardiovasc Surg 1999;118:968-9.

16 Neri E, Capannini G, Carone E, et al. Evolution toward dissection of an intramural hematoma of the ascending aorta. Ann Thorac Surg 1999;68: 1855-6.

17 Stanson AW, Kazmier FJ, Hollier LH, et al. Penetrating atherosclerotic ulcers of the thoracic aorta: natural history and clinicopathologic correlations. Ann Vasc Surg 1986;1:15-23.

18 Harris JA, Bis KG, Glover JL, et al. Penetrating atherosclerotic ulcers of the aorta. F Vasc Surg 1994;19:90-9.

19 Ando Y, Minami H, Muramoto H, et al. Rupture of thoracic aorta caused by penetrating aortic ulcer. Chest 1994;106:624-6.

20 Vilacosta I, San Román JA, Aragoncillo P. Atherosclerotic aortic rupture: documentation by transesophageal echocardiography. I Am Soc Echocardiogr (in press)

21 Tisnado J, Cho S, Beachley MC, et al. Ulcerlike projections: a precursor angiographic sign to thoracic aortic dissection. AfR Am f Roentgenol 1980; 135:719-22.

22 Hayashi H, Matsuoka Y, Sakamoto I, et al. Penetrating atherosclerotic ulcer of the aorta: imaging features and disease concept. Radiographics 2000;20: 995-1005.

23 Yucel EK, Steinberg FL, Egglin TK, et al. Penetrating aortic ulcers: diagnosis with MR imaging. Radiology 1990;177:779-81.

24 Movsowitz HD, David M, Movsowitz C, et al. Penetrating atherosclerotic aortic ulcers: the role of transesophageal echocardiography in diagnosis and clinical management. Am Heart f 1993;126:745-7.

25 Atar S, Nagai T, Birnbaum Y, et al. Transesophageal echocardiographic Doppler findings in patients with penetrating aortic ulcers. Am f Cardiol $1999 ; 83: 133-5$.

26 Miller JS, Lemaire SA, Coselli JS. Evaluating aortic dissection: when is coronary angiography indicated? Heart 2000;83:615-6.

27 Shimizu H, Yoshino $\mathrm{H}$, Udagawa $\mathrm{H}$, et al. Prognosis of aortic intramural hemorrhage compared with classic aortic dissection. Am f Cardiol 2000;85: $792-5$. 